\title{
SOME RELATIONS BETWEEN SEMIGROUPS OF POLYHEDRA ${ }^{1}$
}

\author{
ILAN KOZMA
}

Abstract. All spaces are CW-complexes of finite type. The notation "=" means homotopy equivalence. The following theorems are proved:

(1) If $X \vee A=Y \vee A$ then there exists $T$ such that $\Omega X \times T=\Omega Y \times T$,

(2) $X \times A=Y \times A$ implies that there is a $T$ such that $\Sigma X \vee T=$ $\Sigma Y \vee T$.

A partial converse is also proved. As a corollary we get that if $X \vee A=Y \vee A$ then $\pi_{*}(X)=\pi_{*}(Y)$ and if $X \times A=Y \times A$ then $h_{*}(X)=$ $h_{*}(Y)$ for many homology theories.

1. Introduction. We work with the set $W$ of based homotopy equivalence classes of connected $\mathrm{CW}$-complexes of finite type, i.e., complexes with finitely many cells in each dimension. We are deliberately going to confuse a space with its equivalence class. We may also want to consider the subset $W_{1}$ of all 1-connected complexes.

We will denote the one point union by $X+Y$.

We have two semigroup structures on this set, namely $X+Y$ and $X \times Y$; these can be used to define two equivalence classes and Grothendieck groups: $G_{1}=G_{1}(W), G_{2}=G_{2}(W)$, by setting $X \sim_{1} Y$ if and only if there exists an $A$ in $W$ such that $X+A=Y+A$, and $X \sim_{2} Y$ if and only if there exists an $A$ such that $X \times A=Y \times A$.

We have chosen to work with complexes of finite type because of what we need for Corollaries 2.11 and 2.12. If we want to pass to a larger class of spaces, some care has to be taken, because for any $X$ and $Y, X+A=$ $Y+A$ if we set $A=\left(+_{i=1}^{\infty} X\right)+\left(+_{i=1}^{\infty} Y\right)$. Similarly $X \sim_{2} Y$.

These equivalence relations have been investigated by a number of people; among them: Freyd ([2], [3]), Hilton ([4], [5]), Hilton-Roitberg [7]. An exposition of Freyd's work can be found in Cohen [1].

Freyd worked in the stable range and proved many interesting results there about $G_{1}$. Among other things he completely determined the structure

Received by the editors September 24, 1972.

AMS (MOS) subject classifications (1970). Primary 55D10, 55D35, 55D40, 55E20.

Key words and phrases. Noncancellation, suspension, one point union, loop spaces, product, homotopy groups, homology groups.

${ }^{1}$ This work is part of the author's doctoral dissertation, written at Cornell University under Professor P. J. Hilton. 
of $G_{1}$ for stable compact polyhedra, and gave the first example of two spaces $X, Y$ such that $X \neq Y$, but $X \sim_{1} Y$ stably. His example can also serve as an example in the nonstable range.

Hilton gives some nonstable examples of the same phenomenon, i.e., $X \nsim_{1} Y$ and $X \neq Y$. He also proves Corollary 2.11 where $X, Y, A$ are assumed to be suspensions, and Corollary 2.12 for ordinary homology.

Hilton-Roitberg considered the second equivalence class and gave a few examples of $X \sim_{2} Y$ with $X \neq Y$. They are especially interested in finitedimensional examples. They produced examples with $X \times S^{3}=Y \times S^{3}$.

As a further nonsimply connected example we prove

PROposition. $\quad L_{p, q} \sim_{1} L_{p, \bar{q}}$ where $L_{p, q}$ denotes a three-dimensional lens space.

Proof. It is well known that $L_{p, q}=P_{p} \cup_{\alpha} e^{3}$ where $P_{p}$ is the pseudo projective plane with fundamental group $Z_{p}$ and $\alpha$ is some map. Similarly, $L_{p, \bar{q}}=P_{p} \cup_{\beta} e^{\prime 3}$. Moreover, $\pi_{2} L_{p, \bar{q}}=\pi_{2} L_{p, q}=0$. So

$$
L_{p, q} \vee S^{3}=P_{p} \cup_{\alpha} e^{3} \vee S^{3}=P_{p} \cup_{\alpha} e^{3} \cup_{\beta} e^{\prime 3}=L_{p, \bar{q}} \vee S^{3} .
$$

It appears certain that the two equivalence classes are different, and Freyd's [2] examples should serve as an example where $X \sim_{1} Y$ but $X \sim_{2} Y$.

Here we are concerned with relations between the two equivalence relations. More specifically, if we denote by $W_{1}$ the subset of $W$ consisting of all 1-connected spaces, then Theorem 2.4 could be formulated as follows: The map $\Omega: W_{1} \rightarrow W$ which sends $X \rightarrow \Omega X$ can be extended to a map (not a homomorphism) $\Omega: G_{1}\left(W_{1}\right) \rightarrow G_{2}(W)$ of the Grothendieck groups. Dually, the map $\Sigma: W \rightarrow W_{1}$ sending $X$ to $\Sigma X$ can be extended to a map $\Sigma: G_{2}(W) \rightarrow$ $G_{1}\left(W_{1}\right)$.

If we denote by $H^{\prime} \subset G_{1}\left(W_{1}\right)$ the subset of all equivalence classes, each of which contains an $H^{\prime}$-space, and $H \subset G_{2}(W)$ the subset of equivalence classes containing $H$-spaces, then Theorems 2.10 and 2.7 state that $\Omega_{\mid H^{\prime}}: H^{\prime} \rightarrow G_{2}(W)$ is injective, and that $\Sigma_{\mid H}: H \rightarrow G_{1}\left(W_{1}\right)$ is injective.

From Theorems 2.1 and 2.4 we can easily see that if $X \sim_{1} Y$ in $W_{1}$ or $X \sim_{2} Y$ in $W$, then they have the same homology and homotopy groups.

In fact, more is true. If $X \sim_{1} Y$ or $X \sim_{2} Y$, it can be easily shown that $\Gamma_{i}(X) \cong \Gamma_{i}(Y)$, where $\Gamma$ is the Whitehead gamma group [8], that the isomorphisms $\pi_{i}(X) \cong \pi_{i}(Y), H_{i}(X) \cong H_{i}(Y)$ can be chosen to commute with the Hurewicz homomorphism, and that for finite dimensional spaces $H^{*}(X)$ and $H^{*}(Y)$ are isomorphic both as modules over the Steenrod algebra and as rings.

I wish to thank Professor P. Hilton for his advice and encouragement. 
2. Proofs of the theorems. In what follows we will make essential use of the following identities for homotopy types.

(a) $\quad \Sigma(X \times Y)=\Sigma X+\Sigma Y+\Sigma(X \wedge Y)$

[6, p. 104],

$$
\Omega(X+Y)=\Omega X \times \Omega Y \times \Omega \Sigma(\Omega X \wedge \Omega Y) \quad[6, \text { p. 216]. }
$$

Theorem 2.1. If $X \times A=Y \times A$ and $X, Y, A$ are in $W$, then there exists $T \in W_{1}$ which is also a suspension so that $\Sigma X+T=\Sigma Y+T$.

Proof. Let $\alpha_{1}=\Sigma X, \beta_{1}=\Sigma Y, \alpha_{2}=\Sigma A+\Sigma(X \wedge A), \beta_{2}=\Sigma A+\Sigma(Y \wedge A)$. Then, using $(\alpha)$ and the fact that $\Sigma(X \times A)=\Sigma(Y \times A)$, we get that $\alpha_{1}+$ $\alpha_{2}=\beta_{1}+\beta_{2}$. Define $\alpha_{n}=\Sigma A^{n}+\Sigma\left(X \wedge A^{n}\right), \beta_{n}=\Sigma A^{n}+\Sigma\left(Y \wedge A^{n}\right)$, where $A^{n}$ denotes the $n$-fold smash product. Then we have that:

$$
\begin{aligned}
\alpha_{n}+\alpha_{n+1} & =\Sigma A^{n}+\Sigma\left(X \wedge A^{n}\right)+\Sigma A^{n+1}+\Sigma\left(X \wedge A^{n+1}\right) \\
& =\Sigma A^{n}+A^{n} \wedge(\Sigma X+\Sigma A+\Sigma(X \wedge A)) .
\end{aligned}
$$

Using ( $\alpha$ ) again, we get that the last term is equal to $\beta_{n}+\beta_{n+1}$. Let $T=十_{i=2}^{\infty} \alpha_{i}$. Then $T=+_{i=2}^{\infty} \beta_{i}$, because

Moreover

$$
+_{i=2}^{\infty} \alpha_{i}=+_{i=1}^{\infty}\left(\alpha_{2 i}+\alpha_{2 i+1}\right)=+_{i=2}^{\infty}\left(\beta_{2 i}+\beta_{2 i+1}\right) \text {. }
$$

$$
\begin{aligned}
\Sigma X+T & =+_{i=1}^{\infty} \alpha_{i}=+_{i=1}^{\infty}\left(\alpha_{2 i-1}+\alpha_{2 i}\right)=+_{i=1}^{\infty}\left(\beta_{2 i-1}+\beta_{2 i}\right) \\
& =\underset{i=1}{+} \beta_{i}=\Sigma Y+T .
\end{aligned}
$$

The proofs of the other theorems are similar, though more involved; we need some preliminaries first:

Definition. Let $F$ be the minimal set of functions; $f: W \times W \rightarrow W$ so that:

(a) The function $P_{1} \in F$ where $P_{1}(X, Y)=X$.

The function $f(X)=*$ is in $F$.

(b) The function $P_{2} \in F$ where $P_{2}(X, Y)=Y$.

The function $P_{3} \in F$ where $P_{3}(X, Y)=C$ for some fixed $C \in W$.

(c) If $g, f$ are in $F$ then $f \times g \in F$ where $(f \times g)(X, Y)=f(X, Y) \times g(X, Y)$.

(d) If $f_{1}, \cdots, f_{n}$ are in $F$, then $\Omega \Sigma\left(f_{1} \wedge \cdots \wedge f_{n}\right) \in F$.

So every function in $F$ can be obtained from $P_{1}, P_{2}$, and $P_{3}$ by a sequence of operations $\alpha_{1} \cdots \alpha_{n}$ of type (c) or (d). We will call such a sequence a presentation of $f$. All our constructions in $F$ will actually be constructions on elements $(f, \alpha)$, where $\alpha$ is a presentation of $f$. 
Definition. For any $f \in F, \operatorname{deg} f$ will be defined as follows:

(a) $\operatorname{deg} P_{1}=0, \operatorname{deg} *=\infty$,

(b) $\operatorname{deg} P_{2}=0, \operatorname{deg} P_{3}=0$,

(c) $\operatorname{deg} f \times g=\min \{\operatorname{deg} f, \operatorname{deg} g\}$,

(d) $\operatorname{deg} \Omega \Sigma\left(f_{1} \wedge \cdots \wedge f_{n}\right)=\operatorname{deg} f_{1}+\cdots+\operatorname{deg} f_{n}+(n-1)$.

It is obvious from the definition that, for any $X, Y \in W$ and $f \in F, \operatorname{deg} f \leqq$ connectivity of $f(X, Y)$.

Lemma 2.2. Let $g, f_{1} \cdots f_{n} \in F$. Then

$$
\begin{aligned}
& \Omega \Sigma\left(f_{1} \wedge \cdots \wedge\right. \\
& \left.\quad=\Omega \Sigma\left(f_{n} \times g\right)\right) \\
& \left.\quad=\Omega \cdots \wedge f_{n}\right) \times \Omega \Sigma\left(f_{1} \wedge \cdots \wedge f_{n-1} \wedge g\right) \times \alpha
\end{aligned}
$$

where $\alpha \in F$ and

$$
\operatorname{deg} \alpha>\max \left\{\operatorname{deg} \Omega \Sigma\left(f_{1} \wedge \cdots \wedge f_{n}\right), \operatorname{deg} \Omega \Sigma\left(f_{1} \wedge \cdots \wedge f_{n-1} \wedge g\right)\right\} .
$$

Proof. To prove this we use the identities $(\alpha)$ and $(\beta)$. Write $f$ for $f_{1} \wedge \cdots \wedge f_{n-1}$. Then

$$
\begin{aligned}
& \Omega \Sigma\left(f \wedge\left(f_{n} \times g\right)\right)=\Omega\left(\Sigma f \wedge f_{n}+\Sigma f \wedge g+\Sigma f \wedge f_{n} \wedge g\right) \\
& =\Omega \Sigma\left(f \wedge f_{n}\right) \times \Omega \Sigma(f \wedge g) \times \Omega \Sigma\left(f \wedge f_{n} \wedge g\right) \\
& \quad \times \Omega \Sigma\left(\left(\Omega \Sigma f \wedge f_{n}\right) \wedge(\Omega \Sigma f \wedge g)\right) \\
& \quad \times \Omega \Sigma\left(\left[\Omega \Sigma\left(f \wedge f_{n}\right) \times \Omega \Sigma(f \wedge g) \times \Omega \Sigma\left(\left(\Omega \Sigma f \wedge f_{n}\right) \wedge(\Omega \Sigma f \wedge g)\right)\right]\right.
\end{aligned}
$$

$\left.\wedge \Omega \Sigma\left(f \wedge f_{n} \wedge g\right)\right)$.

Hence $\alpha$ is defined by the previous equation, and obviously has the right properties.

Theorem 2.3. Let $P, Q, B$ be in $W$, and $\psi, \varphi \in F$, with $\operatorname{deg} \varphi>0$, such that $P \times B \times \varphi(P, B)=Q \times B \times \varphi(Q, B)$. Then there exists $T \in W$ such that $\psi(P, B) \times T=\psi(Q, B) \times T$.

As an immediate corollary we have:

Theorem 2.4. Let $X, Y, A$ be in $W_{1}$ satisfying $X+A=Y+A$. Then there exists $T \in W$ such that $\Omega X \times T=\Omega Y \times T$.

Proof. Use $(\beta)$ to get

$$
\Omega X \times \Omega A \times \Omega \Sigma(\Omega X \wedge \Omega A)=\Omega Y \times \Omega A \times \Omega \Sigma(\Omega Y \wedge \Omega A) .
$$

The theorem will follow from Theorem 2.3 by putting $P=\Omega X, Q=\Omega Y$, $B=\Omega A, \varphi=\Omega \Sigma\left(P_{1} \wedge P_{2}\right)$, and $\psi=P_{1}$.

Definition AND Lemma 2.5. For any $f \in F$ we will define $\bar{f} \in F$. If $P, Q$, $B$ satisfy the assumptions of Theorem 2.3 , we will have $f(P, B) \times \bar{f}(P, B)=$ $f(Q, B) \times \bar{f}(Q, B)$. Moreover, $\operatorname{deg} f \leqq \operatorname{deg} \bar{f}$. 
Proof. The definition and the proof will be by induction on the length of the presentation of $f$ :

(a) $\bar{P}_{1}=P_{2} \times \varphi, \bar{*}=*$. Both claims about $f$ follow directly from the assumptions of Theorem 2.3.

(b) $\bar{P}_{2}=* . \bar{P}_{3}=*$.

(c) Suppose $\bar{f}_{1}$ and $\bar{f}_{2}$ are defined. Define $\overline{f_{1} \times f_{2}}=\bar{f}_{1} \times \bar{f}_{2}$. Again, the verification is immediate.

(d) Suppose $\bar{f}_{1}, \cdots, \bar{f}_{n}$ are defined. Let $f=\Omega \Sigma\left(f_{1} \wedge \cdots \wedge f_{n}\right)$. Then by Lemma 2.2 and induction on $n$, we get

$$
\Omega \Sigma\left[\left(f_{1} \times \bar{f}_{1}\right) \wedge \cdots \wedge\left(f_{n} \times \bar{f}_{n}\right)\right]=\left(\prod \Omega \Sigma\left(f_{1}^{\varepsilon_{1}} \wedge \cdots \wedge f_{n}^{\varepsilon_{n}}\right)\right) \times \beta
$$

where $\varepsilon_{i}=0,1$ and by definition $f^{0}=f, f^{1}=\bar{f}$, the product runs over all possible combinations, and $\beta \in F$ with $\operatorname{deg} \beta>\operatorname{deg} f$. Define $\bar{f}=$ $\left(\prod \Omega \Sigma\left(f_{1}^{\varepsilon_{1}} \cdots f_{n}^{\varepsilon_{n}}\right)\right) \times \beta$ where the product runs over all possible combinations with $\varepsilon_{i}=1$ for at least one $i$. The statement about the degrees is immediate, and the other statement follows from the fact that

$$
\begin{aligned}
\left(\Omega \Sigma\left[\left(f_{1} \times \bar{f}_{1}\right) \wedge \cdots \wedge\left(f_{n} \times \bar{f}_{n}\right)\right]\right)(P, B) & \\
= & \left(\Omega \Sigma\left[\left(f_{1} \times \bar{f}_{1}\right) \wedge \cdots \wedge\left(f_{n} \times \bar{f}_{n}\right)\right]\right)(Q, B) .
\end{aligned}
$$

For any $f \in F$ define $f^{0}=f$ and $f^{n}=\overline{f^{n-1}}$. Then:

LEMMA 2.6. For any $f \in F$ so that $f \neq *$, there exists $n>0$ such that $\operatorname{deg} f^{n}>\operatorname{deg} f$.

Proof. The proof is again by induction:

(a) We have that $P_{1}^{2}=\bar{\varphi}$. Hence $n=2$ since $\operatorname{deg} \varphi \geqq 1$.

(b) As $\bar{P}_{2}=*$ and $\bar{P}_{3}=*$, we have that $n=1$.

(c) If $\operatorname{deg} f^{n}>\operatorname{deg} f$ and $\operatorname{deg} g^{m}>\operatorname{deg} g$, then $\operatorname{deg}(f \times g)^{\max (n, m)}>$ $\operatorname{deg}(f \times g)$.

(d) Suppose the assertion is true for $f_{1}, \cdots, f_{n}$, i.e., there exists $m$ such that $\operatorname{deg} f_{i}^{m}>\operatorname{deg} f_{i}$ for $i \leqq n$. Let $f=\Omega \Sigma\left(f_{1} \wedge \cdots \wedge f_{n}\right)$. By induction on $k$ one gets that $f^{k}=\prod \Omega \Sigma\left(f_{1}^{\varepsilon_{1}} \wedge \cdots \wedge f_{n}^{\varepsilon_{n}}\right) \times \gamma$, where all the elements in the product satisfy $\varepsilon_{1}+\cdots+\varepsilon_{n} \geqq k$, and $\gamma \in F$ satisfies $\operatorname{deg} \gamma>\operatorname{deg} f$. If we take $k=n m$, then each element $\Omega \Sigma\left(f_{1}^{\varepsilon_{1}} \wedge \cdots \wedge f_{n}^{\varepsilon_{n}}\right)$ in the product must have at least one $\varepsilon_{i}$ satisfying $\varepsilon_{i} \geqq m$. That will give us that $\operatorname{deg} f^{k}>\operatorname{deg} f$.

Proof of Theorem 2.3. Consider the infinite product $\prod_{i \geqq 0} \psi^{i}(P, B)$. The product is in $W$ because $\operatorname{deg} \psi^{i} \rightarrow \infty$ by Lemma 2.6. Then by Lemma 2.5:

$$
\begin{aligned}
\prod_{i \geqq 0} \psi^{i}(P, B) & =\prod_{i \geqq 0}\left(\psi^{2 i} \times \psi^{2 i+1}\right)(P, B) \\
& =\prod_{i \geqq 0}\left(\psi^{2 i} \times \psi^{2 i+1}\right)(Q, B)=\prod_{i \geqq 0} \psi^{i}(Q, B) .
\end{aligned}
$$


Moreover,

$$
\begin{aligned}
\prod_{i \geqq 1} \psi^{i}(P, B) & =\left(\prod_{i \geqq 1} \psi^{2 i-1} \times \psi^{2 i}\right)(P, B) \\
& =\prod_{i \geqq 1}\left(\psi^{2 i-1} \times \psi^{2 i}\right)(Q, B)=\prod_{i \geqq 1} \psi^{i}(Q, B) .
\end{aligned}
$$

Putting $T=\prod_{i \geqq 1} \psi^{i}(P, B)$ we get the result.

We will turn now to a partial converse of Theorem 2.1.

THEOREM 2.7. If $X, Y$ are connected $H$-spaces of finite type and if there exists $T \in W_{1}$ such that $\Sigma X+T=\Sigma Y+T$, then there exists $A \in W$ such that $X \times A=Y \times A$.

Proof. Use $(\beta)$ to get $\Omega \Sigma X \times \Omega T \times \Omega \Sigma(\Omega \Sigma X \wedge \Omega T)=\Omega \Sigma Y \times \Omega T \times$ $\Omega \Sigma(\Omega \Sigma Y \wedge \Omega T)$.

By a theorem of Sugawara [6, p. 208], $\Omega \Sigma X=X \times \Omega \Sigma(X \wedge X)$ for any $H$-space $X$. The theorem now follows from Theorem 2.3 if we take $P=X$, $Q=Y, B=\Omega T$, and $\varphi=\Omega \Sigma\left(P_{1} \wedge P_{1}\right) \times \Omega \Sigma\left(P_{2} \wedge \Omega \Sigma P_{1}\right)$ and $\psi=P_{1}$.

We will also prove a partial converse to Theorem 2.4. We need two preparatory lemmas.

Lemma 2.8. Let $X, Y \in W_{1}, T \in W$. If $\Omega X \times T=\Omega Y \times T$ then for every $M \in W_{1}$ there exists $M^{\prime} \in W$ such that $\Omega(X+M) \times M^{\prime}=\Omega(Y+M) \times M^{\prime}$.

Proof. Using $(\beta)$ it is enough to prove that there exists $M^{\prime \prime}$ such that $\Omega \Sigma(\Omega X \wedge \Omega M) \times M^{\prime \prime}=\Omega \Sigma(\Omega Y \wedge \Omega M) \times M^{\prime \prime}$. But that will follow from Theorem 2.3 by setting $P=\Omega X, Q=\Omega Y, B=T, \varphi=*, \psi=\Omega \Sigma\left(P_{1} \wedge P_{3}\right)$ and $C=\Omega M$.

The following result is known to Ganea. We give a proof in the spirit of the arguments of this paper.

LEMMA 2.9. If $X$ is a simply connected $H^{\prime}$-space of finite type, then there exists a suspension $A$ of finite type such that $X+A$ is a suspension.

Proof. It is shown in [6, p. 209] that if $X$ is an $H^{\prime}$-space, then the standard map $r: \Sigma \Omega X \rightarrow X$ has a cross section $i: X \rightarrow \Sigma \Omega X$ and then $X+X_{1}=$ $\Sigma \Omega X$ where $X_{1}=\Sigma \Omega X \mid X$. Then $X_{1}$ is an $H^{\prime}$-space of finite type, being a retract of $\Sigma \Omega X$. Moreover, conn $X_{1}>$ conn $X$, since the map $r: \Sigma \Omega X \rightarrow X$ induces isomorphism of the first nonvanishing homotopy groups. Iterating the process we construct a sequence of $H^{\prime}$-spaces $X_{i}$ of finite type such that $X_{0}=X, X_{i}+X_{i+1}=\Sigma \Omega X_{i}$, and conn $X_{i+1}>\operatorname{conn} X_{i}$. If we set $A=+_{i=1}^{\infty} X_{i}$, it is easy to see by arguments already used in the proof of Theorems 2.1 and 2.3 that both $A$ and $X+A$ are suspensions, and that $A$ is of finite type.

THEOREM 2.10. If $X, Y$ are 1-connected $H^{\prime}$-spaces and $\Omega X \times T=\Omega Y \times T$ for some $T \in W$ then there exists $A \in W_{1}$ which is a suspension such that $X+A=Y+A$. 
Proof. First assume $X, Y$ are suspensions: $X=\Sigma X^{\prime}, Y=\Sigma Y^{\prime}$. Apply $(\alpha)$ to get $\Sigma \Omega \Sigma X^{\prime}+\left(\Sigma \Omega \Sigma X^{\prime}\right) \wedge T+\Sigma T=\Sigma \Omega \Sigma Y^{\prime}+\left(\Sigma \Omega \Sigma Y^{\prime}\right) \wedge T+\Sigma T$. Using a theorem of James [9, p. 134] which asserts that $\Sigma \Omega \Sigma X^{\prime}=+_{i=1}^{\infty} \Sigma\left(X^{\prime}\right)^{i}$, and applying the same methods of Theorem 2.1 we get the theorem.

We now consider the general case. By Lemma 2.9, there exist $X^{\prime}, Y^{\prime}$ which are simply connected suspensions of finite type such that $X+X^{\prime}$, $Y+Y^{\prime}$ are suspensions. Set $M=X^{\prime}+Y^{\prime}$. By using Lemma 2.8 , we have that $\Omega(X+M) \times T^{\prime}=\Omega(Y+M) \times T^{\prime}$ for some $T^{\prime} \in W$. As $X+M, Y+M$ are suspensions, we can apply what we have already proved to get $(X+M)$ $+A^{\prime}=(Y+M)+A^{\prime}$ for some $A^{\prime}$.

Corollary 2.11. If $X+A=Y+A$ with $X, Y, A$ simply connected of finite type then $\pi_{*}(X) \cong \pi_{*}(Y)$.

Proof. This is an easy corollary of Theorem 2.4 , since there is cancellation for finitely generated abelian groups.

COROLlaRY 2.12. If $h$ is a homology theory with $h_{*}$ (point) of finite type, or $h_{*}$ (point) a P.I.D. and if $X \times A=Y \times A$ with $X, Y, A$ connected of finite type, then $h_{*}(X) \cong h_{*}(Y)$.

Proof. The assumption on $h_{*}$ establishes that $h(X)$ is of finite type if $X$ is of finite type, or that there is cancellation over $h_{*}$ (point). The conclusion follows using Theorem 2.1.

Note that in the preceding theorem we did not use any Künneth theorem as was done in [4].

\section{REFERENCES}

1. J. Cohen, Stable homotopy, Lecture Notes in Math., vol. 165, Springer-Verlag, Berlin and New York, 1970. MR 42 \#8486.

2. P. Freyd, Stable homotopy, Proc. Conference on Categorical Algebra (La Jolla, Calif., 1965), Springer-Verlag, New York, 1966, pp. 121-172. MR 35 \#2280.

3. - The Grothendieck group for stable homotopy is free, Bull. Amer. Math. Soc. 73 (1967), 84-86. MR 34 \#5086.

4. P. J. Hilton, On the homotopy type of compact polyhedra, Fund. Math. 61 (1967), 105-109. MR 37 \#3556.

5. - Some remarks concerning the semi-ring of polyhedra, Bull. Soc. Math. Belg. 19 (1967), 277-288. MR 37 \#3558.

6. - Homotopy theory and duality, Gordon and Breach, New York, 1965. MR 33 \#6624.

7. P. J. Hilton and J. Roitberg, On principal $S^{3}$ bundles over spheres, Ann. of Math. (2) 90 (1969), 91-107. MR 39 \#7624.

8. J. H. C. Whitehead, A certain exact sequence, Ann. of Math. (2) 52 (1950), 51-110. MR 12, 43.

9. J. Milnor, On the construction FK, Algebraic-Topology, J. F. Adams (Editor), Cambridge Univ. Press, New York, 1972.

Department of Mathematics, University of Chicago, Chicago, Illinois 60637 